\title{
Management of Pancreatic Calculi: An Update
}

\author{
Manu Tandan ${ }^{1}$, Rupjyoti Talukdar ${ }^{1,2}$, and Duvvur Nageshwar Reddy ${ }^{1}$ \\ ${ }^{1}$ Asian Institute of Gastroenterology and ${ }^{2}$ Asian Healthcare Foundation, Hyderabad, India
}

Pancreatolithiasis, or pancreatic calculi (PC), is a sequel of chronic pancreatitis (CP) and may occur in the main ducts, side branches or parenchyma. Calculi are the end result, irrespective of the etiology of $\mathrm{CP}$. $\mathrm{PC}$ contains an inner nidus surrounded by successive layers of calcium carbonate. These calculi obstruct the pancreatic ducts and produce ductal hypertension, which leads to pain, the cardinal feature of $\mathrm{CP}$. Both endoscopic therapy and surgery aim to clear these calculi and decrease ductal hypertension. In small PC, endoscopic retrograde cholangiopancreatography (ERCP) followed by sphincterotomy and extraction is the treatment of choice. Large calculi require fragmentation by extracorporeal shock wave lithotripsy (ESWL) prior to their extraction or spontaneous expulsion. In properly selected cases, ESWL followed by ERCP is the standard of care for the management of large PC. Long-term outcomes following ESWL have demonstrated good pain relief in approximately $60 \%$ of patients. However, ESWL has limitations. Per oral pancreatoscopy and intraductal lithotripsy represent techniques in evolution, and in current practice their use is limited to centers with considerable expertise. Surgery should be offered to all patients with extensive PC, associated multiple ductal strictures or following failed endotherapy. (Gut Liver 2016;10:873-880)

Key Words: Chronic, pancreatitis; Pancreatic calculi; Cholangiopancreatography, endoscopic retrograde; Extracorporeal shockwave lithotripsy

\section{INTRODUCTION}

Chronic pancreatitis (CP) is a disease of diverse etiology characterized by progressive and irreversible changes in the pancreas, resulting in loss of exocrine and endocrine functions. Pain, either continuous or episodic, is the dominant and distressing feature of this illness and significantly worsens the quality of life. Alcohol, smoking, genetic factors, metabolic disturbances and defects in immunity are some of the known etiological agents. ${ }^{1}$ While alcohol is the commonest etiological agent in most industrialized countries, the nonalcoholic idiopathic type of $\mathrm{CP}$ is more prevalent in some countries. ${ }^{2-4}$ Pancreatic calculi (PC) are the sequelae of CP and can occur in about 50\% of patients. $^{5}$ These calculi aggravate or produce the typical pancreatic pain experienced by patients, by obstructing pancreatic ducts and producing upstream ductal hypertension and subsequent parenchymal hypertension. Therapy, either endoscopic or surgical aims at clearing these calculi and reducing the ductal hypertension, relieving pain and improving quality of life. PC seen in the nonalcoholic, idiopathic variety of $\mathrm{CP}$ tend to be large and denser than those seen in the alcoholic variety.,

In this review we will discuss the various aspects of PC with special emphasis on extracorporeal shock wave lithotripsy (ESWL) and endoscopic management.

PCs are classified on the basis of type, numbers and location. They may be (1) radio opaque, radiolucent or mixed; (2) single or multiple; (3) located in the main pancreatic duct (MPD), side branches or in the pancreatic parenchyma; and (4) located in head, body or tail regions (Fig. 1). Majority of PCs are radio opaque while a few are radiolucent or mixed. ${ }^{2}$

\section{PATHOGENESIS AND COMPOSITION}

Pancreatic stone protein (PSP) plays a key role in the formation of PC. ${ }^{8}$ Various factors including gene expression, cause a reduction in PSP. Reduction in PSP results in supersaturation of calcium carbonate in the pancreatic juice. This calcium carbonate is then deposited over an inner nidus. Irrespective of the etiology of $\mathrm{CP}$, the structure and composition of PC are the same suggesting a common pathway for pancreatolithiasis. ${ }^{9}$

Scanning electron microscopy and energy dispersive X-ray fluorescence have revealed that all PC have an amorphous ni-

Correspondence to: Manu Tandan

Asian Institute of Gastroenterology, 6-3-661, Somajiguda, Hyderabad 500 082, India

Tel: +91-40-23378888, Fax: +91-40-23324255, E-mail: mantan_05@rediffmail.com

Received on October 30, 2015. Accepted on December 17, 2015.

pISSN 1976-2283 eISSN 2005-1212 https://doi.org/10.5009/gnl15555

@ This is an Open Access article distributed under the terms of the Creative Commons Attribution Non-Commercial License (http://creativecommons.org/licenses/by-nc/4.0) which permits unrestricted non-commercial use, distribution, and reproduction in any medium, provided the original work is properly cited. 

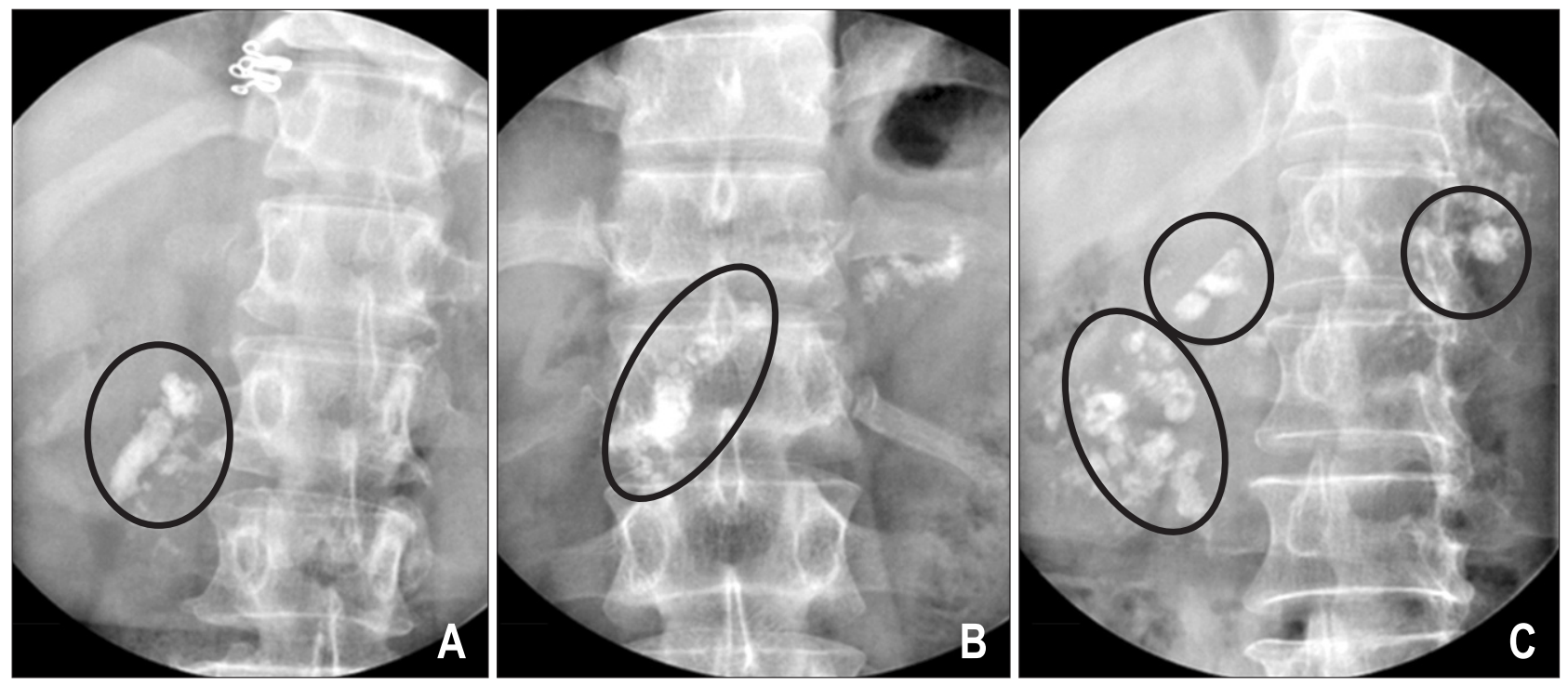

Fig. 1. Pancreatic calculi. (A) Calculi in head. (B) Calculi in head and body. (C) Calculi in head, body and tail.

dus, which forms the center of the PC. The nidus contains elements such as nickel, iron and chromium. It is over this nidus that calcium carbonate in the form of calcite is deposited in multiple layers and over multiple stages. ${ }^{9}$

\section{MODALITIES OF TREATMENT}

As mentioned earlier pain is the dominant symptom in patients with $\mathrm{CP}$ and calculi contribute by obstructing the pancreatic ducts and increasing upstream hypertension. A coexisting ductal stricture can exacerbate the preexisting hypertension.

The following are the modalities of therapy for removal of PC.

(1) Endoscopic therapy: this includes endoscopic retrograde cholangiopancreatography (ERCP) and stone extraction and ESWL. Pancreatoscopy and intraductal lithotripsy as well as and mechanical lithotripsy are lesser-utilized endoscopic techniques for PC extraction.

(2) Surgical therapy: both drainage and resection procedures are widely used in the surgical management of $\mathrm{CP}$ with calculi.

(3) Dissolution of PC: even though chemical agents such as trimethadione have been earlier shown to dissolve stones, these are seldom used in modern day practice.

\section{Endoscopic therapy of PC}

Small and floating calculi, $<5 \mathrm{~mm}$ can be extracted by the standard technique of ERCP and pancreatic sphincterotomy (PS) followed by balloon trawl or basket. However stones $>5 \mathrm{~mm}$ in size are often impacted and difficult to extract by the above mentioned standard technique. , $, 6,10$ These calculi need to be fragmented or pulverized to facilitate their extraction. The European Society of Gastrointestinal Endoscopy (ESGE) clinical guidelines state that endoscopic attempts at MPD stone extraction without prior fragmentation are plagued with low success and relatively high morbidity. ${ }^{11}$ These guidelines also state that for uncomplicated and painful CP with calculi $>5 \mathrm{~mm}$ in the MPD, ESWL should be the first step followed by extraction of the stone fragments at a subsequent ERCP. ${ }^{11}$

The use of Dormia baskets and mechanical lithotripsy following PS for large MPD stones has not been universally successful. A retrospective analysis of over 100 patients showed a success rate of 9\% with the use of Dormia basket. ${ }^{12}$ In a large multicenter study the complication rate with mechanical lithotripsy was unacceptably high, thrice that when compared to biliary stone extraction. ${ }^{13}$ ERCP directed lithotripsy techniques are far less successful and often more risky when compared to those applied in the bile duct because the stones seen in chronic $\mathrm{CP}$ are often hard and also because of the natural tortuosity of the PD. Lithotripsy under direct visualization is more likely to be successful and will be discussed subsequently.

\section{Extracorporeal shock wave lithotripsy}

ESWL was first introduced in the 1980's for the fragmentation of renal and ureteric calculi. ${ }^{14}$ This application was quickly expanded to fragmentation of biliary and PC. ${ }^{15,16}$ It is now the accepted standard of care for the management of large PC at major centers all over the world.,17-24

\section{1) Principle of ESWL}

ESWL is based on the principle of shock wave energy. When energy is released in an enclosed space shock waves are generated. The passage of these shock waves through substances of different acoustic impedance generate compressive stresses on the boundary on the outer surface of these objects. This stress overcomes the tensile strength of targeted object (PC in 


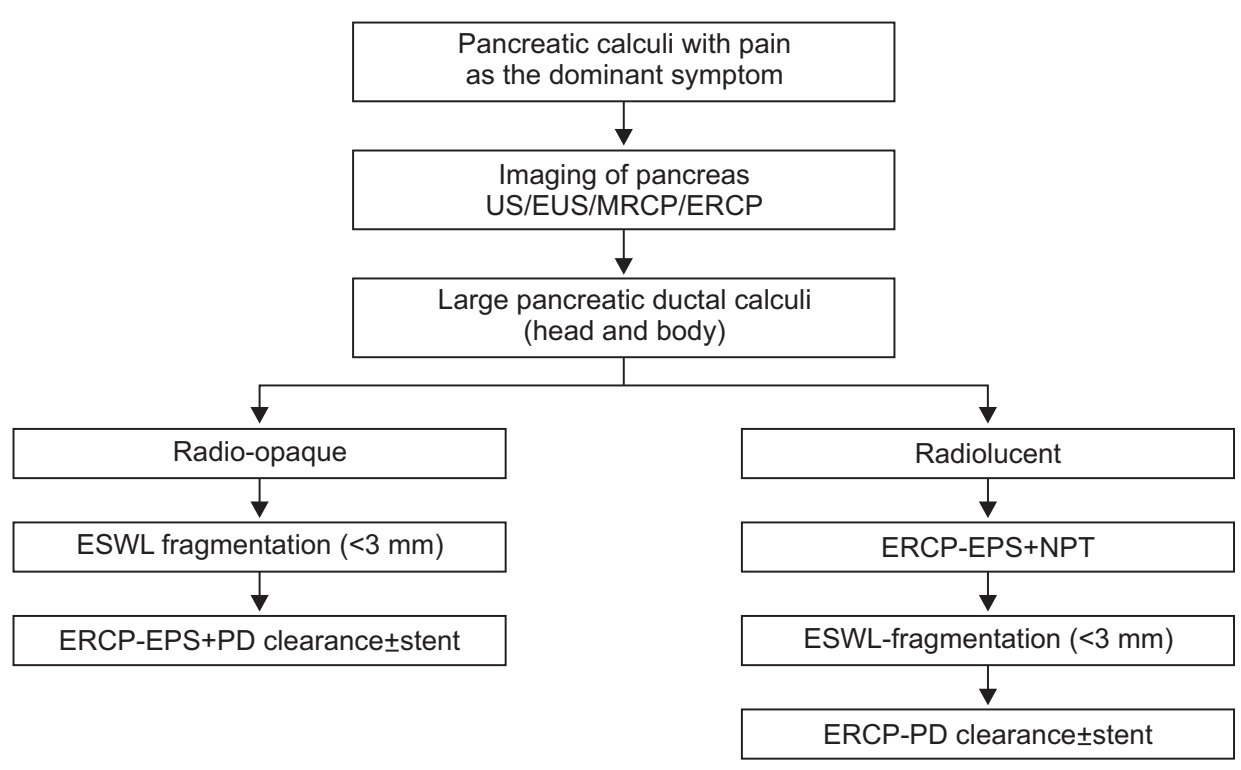

Fig. 2. Protocol for extracorporeal shockwave lithotripsy of large pancreatic duct calculi. ${ }^{26}$

US, ultrasound; EUS, endoscopic ultrasound; MRCP, magnetic resonance cholangiopancreatography; ERCP, endoscopic retrograde cholangiopancreatography; ESWL, extracorporeal shock wave lithotripsy; EPS, endoscopic pancreatic sphincterotomy; PD, pancreatic duct; NPT, naso-pancreatic tube.

Table 1. Efficacy of Extracorporeal Shockwave Lithotripsy for Pancreatic Calculi ${ }^{26}$

\begin{tabular}{lcccc}
\hline \multicolumn{1}{c}{ Author } & No. of patients & Complete clearance, $\%$ & Pain relief, \% & Follow-up, mo \\
\hline Delhaye et al. ${ }^{17}$ & 123 & 59 & 85 & 72 \\
Costamagna et al. $^{19}$ & 35 & 74 & 80 & 27 \\
Kozarek et al. $^{20}$ & 40 & - & 48 & 30 \\
Farnbacher et al. $^{12}$ & 125 & 64 & 55 & 29 \\
Dumonceau et al. $^{18}$ & 29 & - & 76 & 51 \\
Adamek et al. $^{33}$ & 80 & - & 84 & 40 \\
Tandan et al. $^{2}$ & 1,006 & 76 & & 6 \\
\hline
\end{tabular}

this case) and the anterior surface of the calculi crumbles with this impact. Shock waves pass through the calculi and are also reflected from the posterior surface resulting in further fragmentation. ${ }^{25,26}$ The present day lithotripters consist of an electromagnetic shock wave generator, a focusing system for targeting the calculi, and a coupling device. Localization of the calculi is done either by fluoroscopy or ultrasound technique.

\section{2) Indications for ESWL in patients with PC}

ESWL is indicated in all patients of uncomplicated CP with large painful PC not amenable to routine endotherapy and extraction. $^{2,11,26}$ The aim is to fragment the calculi to $<3 \mathrm{~mm}$ in size or to demonstrate a decrease in stone density or heterogenicity of the stones, which occupy the MPD or the side branches. ${ }^{2,17}$

ESWL should not be performed with extensive calculi in head, body and tail. Isolated tail calculi are not targeted at ESWL because of greater chance of collateral damage to the spleen. Patients with multiple strictures, head mass under evaluation, ascites or coexistent pseudocysts are not taken up for ESWL. Any existing cholangitis or coagulopathy due to a concomitant biliary stricture should be treated prior to subjecting the patient to ESWL. ${ }^{2,26}$

\section{3) Protocol of ESWL}

A standard protocol is followed at our center for management of large PC by ESWL and ERCP (Fig. 2).

ESWL is ideally performed with a third generation lithotripter, which uses electromagnetic shock wave for generating shock waves. A maximum of 5,000 to 6,000 shocks are delivered per session with an intensity of 15 to $16 \mathrm{kV}$ at a frequency of 90 shocks per minute. The procedure is carried out on successive days till the desired fragmentation is achieved. ${ }^{26}$ At our center the procedure is performed under epidural anaesthesia (EA). EA, in our experience offers many distinct advantages. ${ }^{27}$ Parenteral sedation, total intra venous analgesia and general anesthesia have been used successfully for ESWL. Lately a mobile or transportable mini lithotripter has been successfully employed for ESWL in some centers. ${ }^{28}$ Another approach that has been recently reported is the use of IV secretin prior to ESWL. The rationale for this approach is to create a fluid-stone interface, akin to lithotripsy for ureteric stones, that results in better stone fragmentation and clearance. ${ }^{29}$

\section{4) Results}

In properly selected cases ESWL is very rewarding procedure 

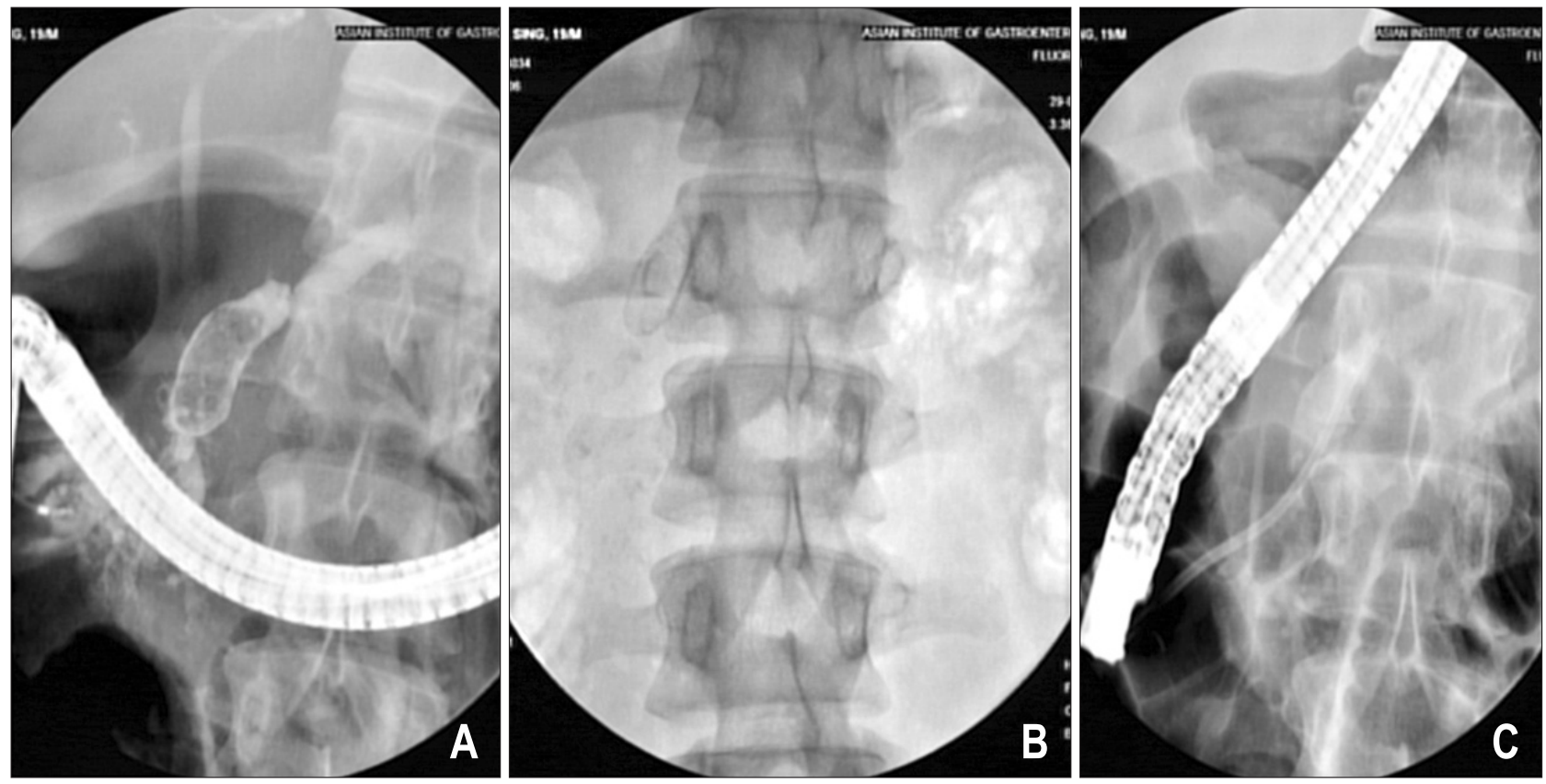

Fig. 3. Extracorporeal shock wave lithotripsy (ESWL) for large pancreatic calculi. ${ }^{26}$ (A) Large calculi in main pancreatic duct with proximal stricture. Pre-ESWL. (B) Post-ESWL calculi pulverized. (C) Fragments cleared and stent placed.

and the results from some of the larger centers are tabulated below (Table 1). In our experience of nearly 2,800 patients with large painful calculi, complete clearance (clearance of $>90 \%$ of stone volume) was achieved in $80 \%$ (Fig. 3), partial clearance (clearance of 50\% to $90 \%$ of stone volume) in $13.8 \%$ while in the rest it was unsuccessful (Fig. 4). ${ }^{30}$

A meta-analysis of 491 patients in 17 studies revealed a clearance rate of $37 \%$ to $100 \%$ with reduction in pain. ${ }^{31}$ Another systematic review of over 1,100 patients described successful stone fragmentation in $89 \%$ of patients. ${ }^{32}$ As CP, especially in the tropics, is a disease of young, the beneficial effects of ESWL on long-term follow-up are important for the procedure to be considered as a standard of care in the management of large PC. Relief between $48 \%$ and $85 \%$ of patients with follow-up between 6 and 51 months has been reported (Table 1). Long-term follow-up following ESWL and endoscopic clearance has been described in a few studies. ${ }^{5,18,21,33,34}$ Pain relief was reported in about $60 \%$ of patients in a few reports. In a recent retrospective analysis of 120 patients on long-term follow-up, partial pain relief was reported in $84 \%$ and complete pain relief with avoidance of narcotic use in 50\% of patients. ${ }^{35}$ Our own experience of long-term follow-up of a maximum of 8 years in over 600 patients, showed a complete pain relief of $60 \%$. Mild or moderate pain was present in $35.7 \%$, while $4 \%$ continued to have severe episodic pain. Pain relief was assessed by decrease in visual analogue scale score, reduction in analgesic use and reduction in number and days of hospitalization. ${ }^{36}$ Though $22.8 \%$ of patients on long-term follow-up developed recurrent calculi in this study, those effected by pain were much fewer in number
Total no. of patients $\quad$ Feb 2004-Feb 2014

\begin{tabular}{ccc}
\multicolumn{3}{c}{2,779} \\
\cline { 2 - 3 } $\begin{array}{c}\text { Complete } \\
\text { Clearance }\end{array}$ & $383(13.8 \%)$ & $170(5.9 \%)$ \\
Partial & Non \\
clearance & fragmented
\end{tabular}

$68 \%$ Three sessions or less for fragmentation $81 \%$ Short term pain relief

$60 \%$ Long-term pain relief (636 patients)

Fig. 4. Extracorporeal shock wave lithotripsy for large pancreatic calculi (experience at the Asian Institute of Gastroenterology, India). ${ }^{30}$

suggesting that not all patients who have recurrent calculi need to be subjected to a repeat endoscopic clearance procedure. The persistence of pain seen in some patients could be a combination of retained or recurrent stones, ongoing active inflammation or other mechanisms of pain production such as ischemia and neural pain.

\section{5) Complications and limitations of ESWL}

ESWL when performed in centers with experience is a safe procedure. Dumonceau et al. ${ }^{11}$ reported morbidity of 5.8\% with a single mortality $(0.05 \%)$ in four large series with over 1,800 patients. A recent study involving 1,470 ESWL procedures reported an overall complication rate of $6.7 \% .{ }^{37}$ In our own experience of 2,800 procedures complications were minimal and mild and could be managed conservatively without the need for any intervention or prolongation of hospital stay., ${ }^{2,631,36}$ These include pain at the site of shock wave delivery, ecchymotic 
patches on skin, occasional abdominal pain and fever. Accurate targeting of the calculi and reduced patient movement decreases the extent of collateral damage and minimizes the complications. ${ }^{2,6,27}$ A few serious and rare compilations have also been described following ESWL. These include perirenal hematoma, biliary obstruction, splenic rupture, bowel perforation, liver trauma as well as necrotising pancreatitis. ${ }^{38-41}$ However these complications are few and occur infrequently.

\section{6) Limitations}

Despite the safety and efficacy, ESWL has its own limitations. The first is the failure of fragmentation seen in a few patients. If these calculi could be identified prior to subjecting them to ESWL then an alternative procedure can be advised. In a recent study Ohyama et $a{ }^{42}$ have shown that calculi with density of $>820.5$ Hounsfield units on noncontrast computed tomography were associated with reduced stone fragmentation and clearance. Further validation of this could help predict therapeutic response to ESWL and avoid the procedure in all those patients where the calculi is less likely to fragment. Another drawback, the recurrence of calculi following ESWL has been well documented. The use of pharmacological agents which can prevent this reformation would be very beneficial in preventing stone recurrence, and reduce the need for repeat interventions. There is also no definite evidence of ESWL and stone clearance improving both exocrine and endocrine dysfunction. A few studies failed to show improvement in both exocrine and endocrine function. ${ }^{21,33}$ Inui et al. ${ }^{23}$ have reported improvement in both endocrine and exocrine functions in their series. In our own experience improvement in endocrine function is seen in a few patients. The numbers are however all too small to arrive at a definitive conclusion. ${ }^{36}$ There are also no reports to suggest that if ESWL is performed early for PC the development of pancreatic carcinoma could be reduced or influenced. Another issue of debate is the practice of ERCP following ESWL. Two uncontrolled studies showed that ESWL alone was adequate in clearing the MPD of calculi. ${ }^{22,23}$ A randomized trial comparing ESWL alone and ESWL followed by ERCP showed equal efficacy in either arm. The treatment cost was higher and a longer hospital stay was seen in patients who had undergone ERCP in addition to ESWL. ${ }^{43}$

\section{Pancreatoscopy and intraductal lithotripsy}

Performance of lithotripsy (electrohydraulic and laser lithotripsy [LL]) under direct vision using pancreatoscopes has considerably improved the armamentarium of the endoscopist for clearing large PC. There is very limited experience in the use of the mother baby cholangioscope (Olympus Medical System, Tokyo, Japan) in the management of pancreatolithiasis and to the best of our knowledge there is no large series or head to head comparison with the newer SPY scope system.

The advent of Spyscope (Spyglass direct visualization system;
Boston Scientific Corp, Natick, MA, USA) for direct visualization of both biliary and pancreatic ductal systems and the concomitant use of newer lasers for lithotripsy has provided a new dimension in the management of pancreatolithiasis. The newly introduced digital Spyglass system should further make the procedures more convenient and efficient.

Per oral pancreatoscopy (POP) and electrohydraulic lithotripsy (EHL) was successfully used in five of the six patients, where PC were cleared in a single session. ${ }^{44}$ POP guided endotherapy was successfully used in 33 patients with EHL and in a further six patients LL was used with a complete stone clearance of 70\%. Adverse effects were reported in $10 \% .{ }^{45} \mathrm{LL}$ can fragment firmer pancreatic stones and offers a potential advantage over EHL. ${ }^{45}$ In a recent retrospective multicentric study of 28 patients some of whom had undergone prior ESWL, POP guided LL using a holmium laser achieved complete stone clearance in 79\% of patients and a partial clearance in a further $11 \%$. A median follow-up of 13 months showed improvement of pain in 25 of 28 patients. Adverse effects were minor and reported in 29\% of patients. ${ }^{46}$ In these studies POP was performed using the Spyglass direct visualization system. The authors suggest that experienced pancreatoscopists should consider POP guided pancreatic stone removal as a viable alternative or a technique complimentary to ESWL. Randomized head to head studies comparing ESWL with POP and lithotripsy are warranted and awaited. At present the experience with EHL and LL using pancreatoscopes is limited to a few centers with considerable expertise. The number of patients are small and long-term follow-up results are awaited. The main disadvantages of POP guided therapy is that it requires non-standard equipment and considerable expertise. Our current opinion is that pancreatoscopy with EHL and LL should be considered as a second option for stones that are refractory to a adequately performed ESWL.

\section{Medical dissolution of pancreatic stones}

A few earlier anecdotal reports showed efficacy of pharmacological agents (trimethadione) for dissolving pancreatic stones. ${ }^{47}$ These reports are not been validated elsewhere and there are no randomized or comparative trails showing their efficacy. ESGE guidelines state that this therapy may have significant side effects and may be attempted in those patients where all other methods have failed and who are not fit surgical candidates. ${ }^{11}$

\section{Role of surgery}

A detailed discussion on surgical techniques and benefits vis-a-vis endotherapy is beyond the scope of this article. Resection, drainage and combined procedure are performed in patients with $\mathrm{CP}$. Even after duodenum preserving resection of head of pancreas, considered the best surgical approach for $\mathrm{CP}$, about 25\% of patients experience recurrence of pain. ${ }^{12}$ Recent studies have compared endotherapy and surgery in CP and conclude that surgery offers the best long-term results. ${ }^{48-51}$ Two 


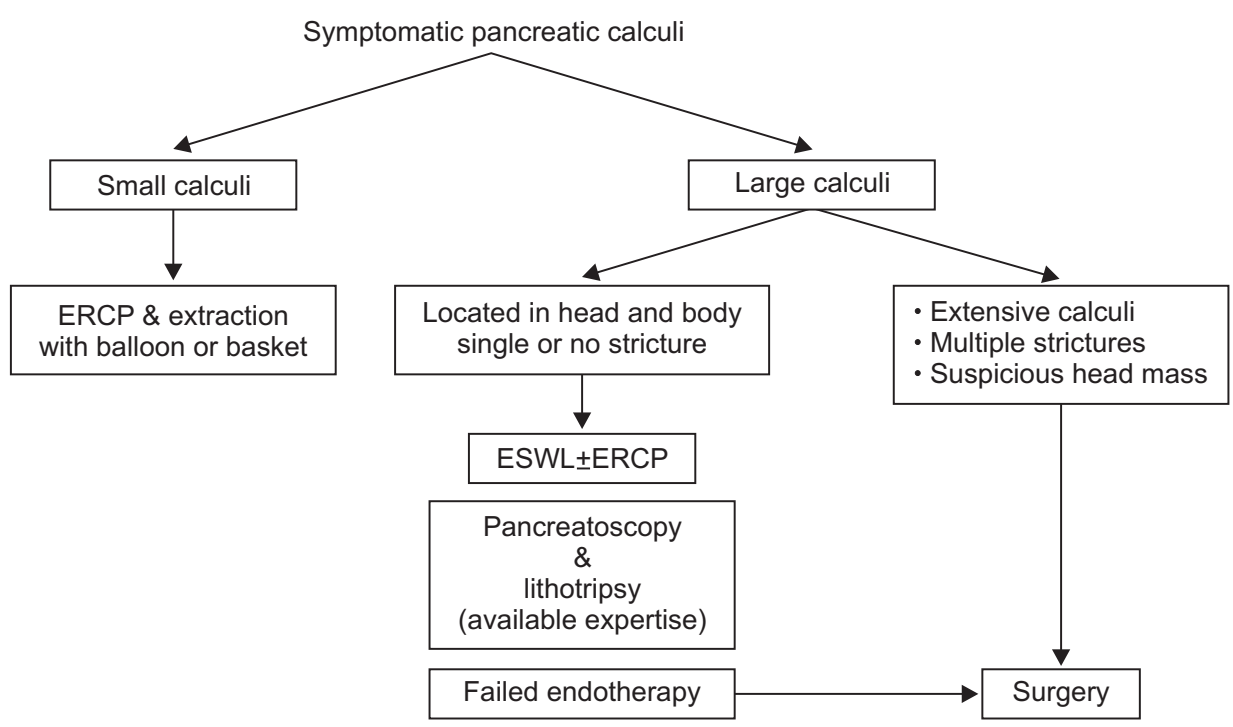

Fig. 5. Algorithm for the management of pancreatic calculi. ERCP, endoscopic retrograde cholangiopancreatography; ESWL, extracorporeal shock wave lithotripsy. of these studies however did not include ESWL as a therapeutic modality and the selection of patients in the endoscopy and surgical arm has been debated. Endotherapy and surgery are complimentary not competitive strategies. Endotherapy should be offered as a first line therapy in patients with uncomplicated PC limited to the head, genu or proximal body, in those patients who do not have multiple strictures or associated head mass. A failure of single or two sessions of endotherapy does not interfere with subsequent surgical management. For patients with extensive calculi, multiple strictures, a suspected pancreatic carcinoma or those who have failed endotherapy, surgery is the better option. An algorithm for endoscopic and surgical management of symptomatic PC is shown in Fig. 5.

\section{CONCLUSIONS}

In conclusion PC are the natural sequel of the ongoing process of $\mathrm{CP}$. Pain is the dominant symptom of patients with $\mathrm{CP}$ and various endoscopy and surgical therapy aims at reducing this pain by eliminating the calculi. Small PC are cleared by the standard technique of PS followed by balloon trawl or basket. Large calculi in uncomplicated patients should be subjected to ESWL for fragmentation prior to a subsequent ERCP. In properly selected patients ESWL is an efficient and useful tool and provides adequate long-term relief. ${ }^{52}$ Spyscopy with intraductal lithotripsy are newer techniques awaiting validation and comparison with ESWL. These procedures should be performed at centers with experience in those patients who do not have successful fragmentation with ESWL. Patients with extensive calculi, multiple strictures, suspicious mass lesions and those who have failed endotherapy are ideal candidates for surgery. Removal of existing PC by any of these above techniques however does not provide answers to many questions. These include recurrence, persistence of pain despite adequate clearance in some patients and the exocrine and endocrine dysfunction, that remain largely unaffected after stone clearance. Further research in these areas is essential in order to provide a final answer to the issues related to PC.

\section{CONFLICTS OF INTEREST}

No potential conflict of interest relevant to this article was reported.

\section{REFERENCES}

1. Lankisch PG, Banks PA. Pancreatitis. New York: Springer-Verlag, 1998.

2. Tandan M, Reddy DN, Santosh D, et al. Extracorporeal shock wave lithotripsy and endotherapy for pancreatic calculi: a large single center experience. Indian J Gastroenterol 2010;29:143-148.

3. Bhasin DK, Singh G, Rana SS, et al. Clinical profile of idiopathic chronic pancreatitis in North India. Clin Gastroenterol Hepatol 2009;7:594-599.

4. Balakrishnan V, Unnikrishnan AG, Thomas V, et al. Chronic pancreatitis: a prospective nationwide study of 1,086 subjects from India. JOP 2008;9:593-600.

5. Rösch T, Daniel S, Scholz M, et al. Endoscopic treatment of chronic pancreatitis: a multicenter study of 1000 patients with longterm follow-up. Endoscopy 2002;34:765-771.

6. Ong WC, Tandan M, Reddy V, Rao GV, Reddy N. Multiple main pancreatic duct stones in tropical pancreatitis: safe clearance with extracorporeal shockwave lithotripsy. J Gastroenterol Hepatol 2006;21:1514-1518.

7. Chari S, Jayanthi V, Mohan V, Malathi S, Madanagopalan N, Viswanathan M. Radiological appearance of pancreatic calculi in tropical versus alcoholic chronic pancreatitis. J Gastroenterol Hepatol 1992;7:42-44. 
8. Jin CX, Naruse S, Kitagawa M, et al. Pancreatic stone protein of pancreatic calculi in chronic calcified pancreatitis in man. JOP 2002;3:54-61.

9. Pitchumoni CS, Viswanathan KV, Gee Varghese PJ, Banks PA. Ultrastructure and elemental composition of human pancreatic calculi. Pancreas 1987;2:152-158.

10. Lehman GA. Role of ERCP and other endoscopic modalities in chronic pancreatitis. Gastrointest Endosc 2002;56(6 Suppl):S237S240.

11. Dumonceau JM, Delhaye M, Tringali A, et al. Endoscopic treatment of chronic pancreatitis: European Society of Gastrointestinal Endoscopy (ESGE) Clinical Guideline. Endoscopy 2012;44:784800.

12. Farnbacher MJ, Schoen C, Rabenstein T, Benninger J, Hahn EG, Schneider HT. Pancreatic duct stones in chronic pancreatitis: criteria for treatment intensity and success. Gastrointest Endosc 2002;56:501-506.

13. Thomas M, Howell DA, Carr-Locke D, et al. Mechanical lithotripsy of pancreatic and biliary stones: complications and available treatment options collected from expert centers. Am J Gastroenterol 2007;102:1896-1902.

14. Chaussy C, Schmiedt E, Jocham D, Brendel W, Forssmann B, Walther V. First clinical experience with extracorporeally induced destruction of kidney stones by shock waves. J Urol 1982;127:417420.

15. Sauerbruch T, Stern M. Fragmentation of bile duct stones by extracorporeal shock waves: a new approach to biliary calculi after failure of routine endoscopic measures. Gastroenterology 1989;96:146-152.

16. Neuhaus H. Fragmentation of pancreatic stones by extracorporeal shock wave lithotripsy. Endoscopy 1991;23:161-165.

17. Delhaye M, Vandermeeren A, Baize M, Cremer M. Extracorporeal shock-wave lithotripsy of pancreatic calculi. Gastroenterology 1992;102:610-620.

18. Dumonceau JM, Devière J, Le Moine 0, et al. Endoscopic pancreatic drainage in chronic pancreatitis associated with ductal stones: long-term results. Gastrointest Endosc 1996;43:547-555.

19. Costamagna G, Gabbrielli A, Mutignani M, et al. Extracorporeal shock wave lithotripsy of pancreatic stones in chronic pancreatitis: immediate and medium-term results. Gastrointest Endosc 1997;46:231-236.

20. Kozarek RA, Brandabur JJ, Ball TJ, et al. Clinical outcomes in patients who undergo extracorporeal shock wave lithotripsy for chronic calcific pancreatitis. Gastrointest Endosc 2002;56:496500.

21. Schneider HT, May A, Benninger J, et al. Piezoelectric shock wave lithotripsy of pancreatic duct stones. Am J Gastroenterol 1994;89:2042-2048.

22. Ohara H, Hoshino M, Hayakawa T, et al. Single application extracorporeal shock wave lithotripsy is the first choice for patients with pancreatic duct stones. Am J Gastroenterol 1996;91:13881394.
23. Inui $\mathrm{K}$, Tazuma $\mathrm{S}$, Yamaguchi $\mathrm{T}$, et al. Treatment of pancreatic stones with extracorporeal shock wave lithotripsy: results of a multicenter survey. Pancreas 2005;30:26-30.

24. Delhaye M, Arvanitakis M, Bali M, Matos C, Devière J. Endoscopic therapy for chronic pancreatitis. Scand J Surg 2005;94:143-153.

25. Grasso M, Spaliviero M. Extracorporeal shockwave lithotripsy [Internet]. Atlanta: WebMD; 2008 [cited 2006 Jun 29]. Available from: http://www.emedicine.com/med/topic3024.htm.

26. Tandan M, Reddy DN. Extracorporeal shock wave lithotripsy for pancreatic and large common bile duct stones. World J Gastroenterol 2011;17:4365-4371.

27. Darisetty S, Tandan M, Reddy DN, et al. Epidural anesthesia is effective for extracorporeal shock wave lithotripsy of pancreatic and biliary calculi. World J Gastrointest Surg 2010;2:165-168.

28. Milovic V, Wehrmann T, Dietrich CF, Bailey AA, Caspary WF, Braden B. Extracorporeal shock wave lithotripsy with a transportable mini-lithotripter and subsequent endoscopic treatment improves clinical outcome in obstructive calcific chronic pancreatitis. Gastrointest Endosc 2011;74:1294-1299.

29. Choi EK, McHenry L, Watkins JL, et al. Use of intravenous secretin during extracorporeal shock wave lithotripsy to facilitate endoscopic clearance of pancreatic duct stones. Pancreatology 2012;12:272-275.

30. Tandan M, Reddy DN. ESWL of large pancreatic calculi: a decades experience. United European Gastroenterol J 2014;2(Suppl 1):A7.

31. Guda NM, Partington S, Freeman ML. Extracorporeal shock wave lithotripsy in the management of chronic calcific pancreatitis: a meta-analysis. JOP 2005;6:6-12.

32. Nguyen-Tang T, Dumonceau JM. Endoscopic treatment in chronic pancreatitis, timing, duration and type of intervention. Best Pract Res Clin Gastroenterol 2010;24:281-298.

33. Adamek HE, Jakobs R, Buttmann A, Adamek MU, Schneider AR, Riemann JF. Long term follow up of patients with chronic pancreatitis and pancreatic stones treated with extracorporeal shock wave lithotripsy. Gut 1999;45:402-405.

34. Delhaye M, Arvanitakis M, Verset G, Cremer M, Devière J. Longterm clinical outcome after endoscopic pancreatic ductal drainage for patients with painful chronic pancreatitis. Clin Gastroenterol Hepatol 2004;2:1096-1106.

35. Seven G, Schreiner MA, Ross AS, et al. Long-term outcomes associated with pancreatic extracorporeal shock wave lithotripsy for chronic calcific pancreatitis. Gastrointest Endosc 2012;75:9971004.e1.

36. Tandan M, Reddy DN, Talukdar R, et al. Long-term clinical outcomes of extracorporeal shockwave lithotripsy in painful chronic calcific pancreatitis. Gastrointest Endosc 2013;78:726-733.

37. Li BR, Liao Z, Du TT, et al. Risk factors for complications of pancreatic extracorporeal shock wave lithotripsy. Endoscopy 2014;46:10921100.

38. Hirata N, Kushida Y, Ohguri T, Wakasugi S, Kojima T, Fujita R. Hepatic subcapsular hematoma after extracorporeal shock wave lithotripsy (ESWL) for pancreatic stones. J Gastroenterol 
1999;34:713-716.

39. Leifsson BG, Borgström A, Ahlgren G. Splenic rupture following ESWL for a pancreatic duct calculus. Dig Surg 2001;18:229-230.

40. Plaisier PW, den Hoed PT. Splenic abscess after lithotripsy of pancreatic duct stones. Dig Surg 2001;18:231-232.

41. Karakayali F, Sevmiş S, Ayvaz I, Tekin I, Boyvat F, Moray G. Acute necrotizing pancreatitis as a rare complication of extracorporeal shock wave lithotripsy. Int J Urol 2006;13:613-615.

42. Ohyama H, Mikata R, Ishihara T, et al. Efficacy of stone density on noncontrast computed tomography in predicting the outcome of extracorporeal shock wave lithotripsy for patients with pancreatic stones. Pancreas 2015;44:422-428.

43. Dumonceau JM, Costamagna G, Tringali A, et al. Treatment for painful calcified chronic pancreatitis: extracorporeal shock wave lithotripsy versus endoscopic treatment: a randomised controlled trial. Gut 2007;56:545-552.

44. Howell DA, Dy RM, Hanson BL, Nezhad SF, Broaddus SB. Endoscopic treatment of pancreatic duct stones using a $10 \mathrm{~F}$ pancreatoscope and electrohydraulic lithotripsy. Gastrointest Endosc 1999;50:829-833.

45. Attwell AR, Brauer BC, Chen YK, Yen RD, Fukami N, Shah RJ. Endoscopic retrograde cholangiopancreatography with per oral pancreatoscopy for calcific chronic pancreatitis using endoscope and catheter-based pancreatoscopes: a 10-year single-center experience. Pancreas 2014;43:268-274.

46. Attwell AR, Patel S, Kahaleh M, Raijman IL, Yen R, Shah RJ. ERCP with per-oral pancreatoscopy-guided laser lithotripsy for calcific chronic pancreatitis: a multicenter U.S. experience. Gastrointest Endosc 2015;82:311-318.

47. Noda A, Shibata T, Hamano H, et al. Trimethadione (troxidone) dissolves pancreatic stones. Lancet 1984;2:351-353.

48. Díte P, Ruzicka M, Zboril V, Novotný I. A prospective, randomized trial comparing endoscopic and surgical therapy for chronic pancreatitis. Endoscopy 2003;35:553-558.

49. Cahen DL, Gouma DJ, Nio Y, et al. Endoscopic versus surgical drainage of the pancreatic duct in chronic pancreatitis. N Engl J Med 2007;356:676-684.

50. Rutter K, Ferlitsch A, Sautner T, et al. Hospitalization, frequency of interventions, and quality of life after endoscopic, surgical, or conservative treatment in patients with chronic pancreatitis. World J Surg 2010;34:2642-2647.

51. Cahen DL, Gouma DJ, Laramée P, et al. Long-term outcomes of endoscopic vs surgical drainage of the pancreatic duct in patients with chronic pancreatitis. Gastroenterology 2011;141:1690-1695.

52. Tandan M, Nageshwar Reddy D. Endotherapy in chronic pancreatitis. World J Gastroenterol 2013;19:6156-6164. 